PROCEEDINGS OF THE

AMERICAN MATHEMATICAL SOCIETY

Volume 130, Number 2, Pages 427-432

S 0002-9939(01)06074-9

Article electronically published on May 23, 2001

\title{
INTERPOLATION IN NEST ALGEBRA MODULES
}

\author{
XIAOXIA ZHANG
}

(Communicated by David R. Larson)

\begin{abstract}
Let $\mathcal{A}$ be a nest algebra and $\mathcal{L}$ at $\mathcal{A}$ its invariant projection (or subspace) lattice. In this paper, using order homomorphisms of $\mathcal{L} a t \mathcal{A}$, we give necessary and sufficient conditions on bounded linear operators $X$ and $Y$ on a Hilbert space to guarantee the existence of an operator $A$ in a certain $\mathcal{A}$-module such that $A X=Y$.
\end{abstract}

\section{INTRODUCTION}

In the study of a reflexive operator algebra $\mathcal{A}$, it is useful to know for which vectors $x$ and $y$ there exists $A \in \mathcal{A}$ satisfying $A x=y$. E.C. Lance initiated the study of such problems and gave necessary and sufficient conditions on vectors $x$ and $y$ to guarantee the existence of an operator $A$ in a given nest algebra $\operatorname{Alg} \mathcal{N}$ such that $A x=y$, where $x$ and $y$ are vectors in a Hilbert space $H$ and $\mathcal{N}$ is a nest. In addition, Lance specified the minimum norm such an operator can have. A. Hopenwasser [4] extended Lance's result to the case where the nest $\mathcal{N}$ is replaced by an arbitrary commutative invariant projection lattice. Munch [8] restricted the operator $A$ to be a Hilbert-Schmidt operator in nest algebra Alg $\mathcal{N}$. In [1] the authors considered the problem of finding $A$ so that $A x=y$ and $A$ is required to be in certain ideals contained in $\operatorname{Alg} \mathcal{N}$. All the results mentioned above are in the case of "vector interpolation".

There is another problem related to the one above: for a given operator algebra $\mathcal{A}$ on a Hilbert space $H$, for which bounded operators $X$ and $Y$ on $H$ does there exist an $A$ in $\mathcal{A}$ satisfying $A X=Y$ ? In [5] the authors solved this problem in nest algebra and gave a sufficient and necessary condition. We call this case operator interpolation. It is clear that the "operator interpolation" problem includes the case of the "vector interpolation" problem. Indeed, if we denote by $x \otimes m^{*}$ the rank-one operator defined by the equation $x \otimes m^{*}(u)=\langle m, u\rangle x$, and if we set $X=x \otimes u^{*}$ and $Y=y \otimes u^{*}$, then the equations $A X=Y$ and $A x=y$ represent the same restriction on $A$.

In this paper, we consider the operator interpolation problem in the case of a nest algebra module. We follow the suggestion hinted in [5] and use the order

Received by the editors March 12, 1998 and, in revised form, October 22, 1998, November 22, 1999, and June 14, 2000.

2000 Mathematics Subject Classification. Primary 47L35.

Key words and phrases. Nest algebra, nest algebra module, operator interpolation, order homomorphism. 
homomorphisms of the invariant projection lattice to solve the operator interpolation problem and give a sufficient and necessary condition in following Theorem 1 , extending the main result in [5].

\section{INTERPOLATION IN NEST ALGEBRA MODULES}

Let $H$ be a separable Hilbert space, $B(H)$ the set of all bounded linear operators on $H$ and $\mathcal{A}$ an algebra in $B(H)$.

First, we give some notations and definitions.

A projection $P \in B(H)$ is invariant under $\mathcal{A}$ if $A P=P A P$ for any $A \in \mathcal{A}$. A closed subspace $S$ of $H$ is invariant under $\mathcal{A}$ if $A S \subseteq S$ for any $A \in \mathcal{A}$. A nest $\mathcal{N}$ is a family of closed subspaces of a Hilbert space $H$ totally ordered by inclusion. $\mathcal{N}$ is complete if it contains (0) and $H$ and contains the join (closed linear span) and meet (intersection) of every subfamily.

Since there is a one-to-one correspondence between closed subspaces and projections, a complete nest is also described as a strongly closed, linearly ordered collection of projections on $H$, containing 0 and the identity.

A nest algebra corresponding a nest $\mathcal{N}$ is the set of all bounded linear operators on $H$ that leave each projection (or subspace) in $\mathcal{N}$ invariant.

If $\mathcal{A}$ is a nest algebra, $\mathcal{L} a t \mathcal{A}$ denotes the invariant projection (or subspace) lattice. In this paper all nests we consider are always complete.

Definition 1. Let $\mathcal{A}$ be a nest algebra. We call $\phi$ an order homomorphism of $\mathcal{L}$ at $\mathcal{A}$ into itself if $E \leq F$ implies $\phi(E) \leq \phi(F)$ for any $E, F \in \mathcal{L}$ at $\mathcal{A}$. Denote

Hom $\mathcal{L} a t \mathcal{A}=\{\phi: \phi$ is an order homomorphism from $\mathcal{L}$ at $\mathcal{A}$ into $\mathcal{L} a t \mathcal{A}\}$

and for any $\phi \in \operatorname{Hom} \mathcal{L} a t \mathcal{A}$,

$$
\mathcal{U}_{\phi}=\left\{G \in B(H): \phi(E)^{\perp} G E=0 \text {, for all } E \in \mathcal{L} a t \mathcal{A}\right\}, \quad \phi \in \operatorname{Hom} \mathcal{L} a t \mathcal{A} .
$$

We know that $\mathcal{U}_{\phi}$ is a weakly closed $\mathcal{A}$-module $([\underline{6]})$.

Definition 2 (see [6]). The weakly closed $\mathcal{A}$-module $\mathcal{U}$ is said to be determined by the order homomorphism $\phi$ if $\mathcal{U}=\mathcal{U}_{\phi}$.

We define

$$
H[\mathcal{U}]=\{\phi \in \operatorname{Hom} \mathcal{L} a t \mathcal{A}: \phi \text { determines } \mathcal{U}\} .
$$

By [6], we know that for a given weakly closed $\mathcal{A}$-module $\mathcal{U}$, there is an element $\eta$ in $H[\mathcal{U}]$ such that $\eta(0)=0$.

First, we introduce a theorem of Douglas [3] and an extension theorem (in [5]).

Theorem $\mathbf{D}$ ([3]). Let $Y$ and $X$ be bounded operators on a Hilbert space $H$. The following statements are equivalent:

(1) $\mathrm{r}\left(Y^{*}\right) \subseteq \mathrm{r}\left(X^{*}\right)$;

(2) $Y^{*} Y \leq \lambda^{2} X^{*} X$, for some $\lambda \geq 0$;

(3) there exists a bounded operator $A$ on $H$ so that $A X=Y$.

Here $\mathrm{r}(T)$ means the range of operator T. Moreover if the above conditions are valid, operator $A$ is unique and:

$$
\begin{aligned}
& \text { (a) }\|A\|^{2}=\inf \left\{u: Y^{*} Y \leq u X^{*} X\right\} \\
& \text { (b) } \operatorname{ker}\left(Y^{*}\right)=\operatorname{ker}\left(A^{*}\right) ; \text { and } \\
& \text { (c) } \mathrm{r}\left(A^{*}\right) \subseteq \mathrm{r}(X)^{-} .
\end{aligned}
$$


Theorem $\mathbf{E}([5])$. Let $H, K$ and $L$ be Hilbert spaces, and let $N$ be a subspace of $H$. Let $A$ and $D$ be operators in $B(N, K)$ and in $B(H, L)$ respectively and suppose that, for every vector $x$ in $N$, we have $\|A x\| \leq k\|D x\|$ for some fixed positive constant $k$. Then there exists an operator $\widetilde{A}$ in $B(H, K)$, such that:

a) $\widetilde{A} x=A x$ for every $x \in N$;

b) $\|\widetilde{A} x\| \leq k\|D x\|$ for every $x \in H$.

Theorem 1. Let $\mathcal{A}$ be a nest algebra, $\mathcal{U}$ a weakly closed $\mathcal{A}$-module, and $\phi$ be in $H[\mathcal{U}]$ satisfying $\phi(0)=0$. Let $X$ and $Y$ be bounded operators on $H$. The following are equivalent:

(1) there exists an operator $A$ in $\mathcal{U}$ such that $A X=Y$.

(2) $\sup \left\{\frac{\left\|(\phi(E))^{\perp} Y f\right\|}{\left\|E^{\perp} X f\right\|}: f \in H, E \in \mathcal{L} a t \mathcal{A}\right\}=k<\infty$.

[We use the convention $0 / 0=0$, when necessary.] Moreover if case (2) holds, we may choose the operator $A$ so that $\|A\|=k$.

Remark. When $\phi$ is an identity homomorphism, then Theorem 1 turns into Theorem 2 in [5].

Proof. (1) $\Longrightarrow(2)$ : Suppose there exists the indicated operator $A$ in $\mathcal{U}$, such that $A X=Y$. For any projections $E$ in $\mathcal{L} a t \mathcal{A}$, and for $f$ in $H$, we have

$$
\begin{aligned}
\left\|\phi(E)^{\perp} Y f\right\| & =\left\|\phi(E)^{\perp} A X f\right\|=\left\|\phi(E)^{\perp} A(I-E+E) X f\right\| \\
& \leq\left\|\phi(E)^{\perp} A E X f\right\|+\left\|\phi(E)^{\perp} A E^{\perp} X f\right\| .
\end{aligned}
$$

Since $\phi(E)^{\perp} A E=0$, for every $E$ in $\mathcal{L} a t \mathcal{A}$, we have

$$
\begin{aligned}
\left\|\phi(E)^{\perp} Y f\right\| & \leq\left\|\phi(E)^{\perp} A E^{\perp} X f\right\| \leq\left\|\phi(E)^{\perp} A\right\| .\left\|E^{\perp} X f\right\| \\
& \leq\|A\|\left\|E^{\perp} X f\right\| .
\end{aligned}
$$

Therefore $\|A\|$ may play the role of the desired constant $k$.

$(2) \Longrightarrow(1)$ : Let $X$ and $Y$ be fixed bounded linear operators. We first consider a slightly simpler case. We show by induction that for an arbitrary finite subnest, $\mathcal{N}_{[n]}=\left\{0, E_{1}, E_{2}, \cdots, E_{n}, I\right\} \subseteq \mathcal{L} a t \mathcal{A}$, under the assumption

$$
\sup \left\{\frac{\left\|(\phi(E))^{\perp} Y f\right\|}{\left\|E^{\perp} X f\right\|}: f \in H, E \in \mathcal{N}_{[n]}\right\}=k<\infty,
$$

there exists an operator $A_{[n]} \in \mathcal{U}_{\phi[n]}$ satisfying $A_{[n]} X=Y$, where

$$
\mathcal{U}_{\phi[n]}=\left\{G \in B(H): \phi(E)^{\perp} G E=0, E \in \mathcal{N}_{[n]}\right\} .
$$

First step. If there are no projections in the subnest other than 0 and $I$, by assumption (2):

if $E=I$, we have $\left\|\phi(I)^{\perp} Y f\right\| \leq\left\|I^{\perp} X f\right\|=0$; hence $Y=\phi(I) Y$;

if $E=0, \mathrm{r}\left(Y^{*}\right) \subseteq \mathrm{r}\left(X^{*}\right)$, we have

$$
\left\|\phi(0)^{\perp} Y f\right\| \leq k\|X f\| \Longrightarrow\|Y f\|^{2} \leq k^{2}\|X f\|^{2} .
$$

It is apparent that $Y^{*} Y \leq k^{2} X^{*} X$. By Theorem D, we have $\mathrm{r}\left(Y^{*}\right) \subseteq \mathrm{r}\left(X^{*}\right)$. By Douglas' theorem, we obtain an operator $B$ such that $B X=Y$. Set $A_{[0]}=\phi(I) B$; obviously, $A_{[0]}$ belongs to

$$
\mathcal{U}_{[0]}=\left\{G \in B(H): \phi(E)^{\perp} G E=0, E=0, E=I\right\},
$$


and $A_{[0]} X=\phi(I) B X=\phi(I) Y=Y$. The fact that $\left\|A_{[0]}\right\|$ can be taken equal to $k$ is a consequence of condition (a) of Theorem D.

Second step. Now, suppose that the statement is true for arbitrary finite subnests of $\operatorname{Lat} \mathcal{A}(=\mathcal{N})$ with no more than $n-1$ nontrivial projections.

Let $\left\{0, E_{1}, E_{2}, \cdots, E_{n}, I\right\}=\mathcal{N}_{[n]}$ be a finite subnest (of $\mathcal{L} a t \mathcal{A}$ ) with $n$ nontrivial projections; correspondingly, there exists $\mathcal{U}_{\phi[n]}$.

Denote

$$
X_{1}=E_{1}^{\perp} X \quad \text { and } \quad Y_{1}=\phi\left(E_{1}\right)^{\perp} Y .
$$

We have for each $j=2,3, \ldots, n$,

$$
X_{1}^{*} E_{j}^{\perp}=\left(E_{1}^{\perp} X\right)^{*} E_{j}^{\perp}=X^{*} E_{1}^{\perp} E_{j}^{\perp}=X^{*} E_{j}^{\perp}
$$

and

$$
Y_{1}^{*} \phi\left(E_{j}\right)^{\perp}=\left(\phi\left(E_{1}\right)^{\perp} Y\right)^{*} \phi\left(E_{j}\right)^{\perp}=Y^{*} \phi\left(E_{1}\right)^{\perp} \phi\left(E_{j}\right)^{\perp}=Y^{*} \phi\left(E_{j}\right)^{\perp} .
$$

In the last equality, we use $\phi\left(E_{1}\right) \leq \phi\left(E_{j}\right)$, since $\phi$ is an order homomorphism.

Consequently for each $j=2,3, \ldots, n$,

$$
\begin{aligned}
\left\|\phi\left(E_{j}\right)^{\perp} Y_{1} f\right\| & =\left\|\phi\left(E_{1}\right)^{\perp} \phi\left(E_{j}\right)^{\perp} Y_{1} f\right\| \\
& =\left\|\phi\left(E_{j}\right)^{\perp} \phi\left(E_{1}\right)^{\perp} Y_{1} f\right\| \\
& =\left\|\phi\left(E_{j}\right)^{\perp} Y f\right\| \\
& \leq k\left\|E_{j}^{\perp} X f\right\|=k\left\|E_{j}^{\perp} E_{1}^{\perp} X f\right\| \\
& =k\left\|E_{j}^{\perp} X_{1} f\right\|[\text { by }(2)] .
\end{aligned}
$$

Thus, it follows that assumption (2) holds for the operators $X_{1}$ and $Y_{1}$ with respect to the subnest $\mathcal{N}_{[n]}^{\prime}=\left\{0, E_{2}, \ldots, E_{n}, I\right\}$. Using the inductive hypothesis, there exists an operator $A^{\prime}$ in $\mathcal{U}_{\phi[n]}^{\prime}$ such that $\left\|A^{\prime}\right\| \leq k$, and $A^{\prime} X_{1}=Y_{1}$, where

$$
\mathcal{U}_{\phi[n]}^{\prime}=\left\{G \in B(H): \phi(E)^{\perp} G E=0, E \in \mathcal{N}_{[n]}^{\prime}\right\}
$$

Since $\phi\left(E_{1}\right)^{\perp} Y=\left(\phi\left(E_{1}\right)^{\perp}\right)^{2} Y=\phi\left(E_{1}\right)^{\perp} Y_{1}$, we have

$$
\phi\left(E_{1}\right)^{\perp} Y=Y_{1}=A^{\prime} X_{1}=A^{\prime} E_{1}^{\perp} X=\phi\left(E_{1}\right)^{\perp} A^{\prime} E_{1}^{\perp} X .
$$

Set $B=\phi\left(E_{1}\right)^{\perp} A^{\prime} E_{1}^{\perp}$. Since the projections in $\mathcal{N}_{[n]}$ all commute with each other, it is clear that $B$ is in

$$
\mathcal{U}_{\phi_{[n]}}=\left\{G \in B(H): \phi(E)^{\perp} G E=0, E \in \mathcal{N}_{[n]}\right\} .
$$

Indeed, (i) if $E=E_{1}$, then

$$
\phi\left(E_{1}\right)^{\perp} B E=\phi\left(E_{1}\right)^{\perp} A^{\prime} E_{1}^{\perp} E_{1}=0 .
$$

Also (ii) if $E=E_{j}$ for each $j=2,3, \ldots, n$, then

$$
\begin{aligned}
\phi\left(E_{j}\right)^{\perp} B E_{j} & =\phi\left(E_{j}\right)^{\perp} \phi\left(E_{1}\right)^{\perp} A^{\prime} E_{1}^{\perp} E_{j} \\
& =\phi\left(E_{1}\right)^{\perp} \cdot\left(\phi\left(E_{j}\right)^{\perp} A^{\prime} E_{j}\right) E_{1}^{\perp}=0 .
\end{aligned}
$$

From above, $B$ is in $\mathcal{U}_{\phi[n]}$. Moreover,

$$
B X=\phi\left(E_{1}\right)^{\perp} A^{\prime} E_{1}^{\perp} X=\phi\left(E_{1}\right)^{\perp} A^{\prime} X_{1}=\phi\left(E_{1}\right)^{\perp} Y
$$

and $\|B\|=\left\|\phi\left(E_{1}\right)^{\perp} A^{\prime} E_{1}^{\perp}\right\| \leq\left\|A^{\prime}\right\| \leq k$. 
Next: $\mathrm{r}\left(Y^{*} \phi\left(E_{1}\right)\right) \subseteq \mathrm{r}\left(Y^{*}\right) \subseteq \mathrm{r}\left(X^{*}\right)$ follows from choosing $E=0$ in part (2). (Since $\phi(0)=0^{\perp}=I$.) Theorem $\mathrm{D}$ then asserts the existence of an operator $A_{0}$ for which $A_{0} X=\phi\left(E_{1}\right) Y$. For any vector $f$, we have

$$
\begin{aligned}
\left\|A_{0} X f\right\|^{2}+\|B X f\|^{2} & =\left\|\phi\left(E_{1}\right) Y f\right\|^{2}+\left\|\phi\left(E_{1}\right)^{\perp} Y f\right\|^{2} \\
& =\|Y f\|^{2} \leq k^{2}\|X f\|^{2}
\end{aligned}
$$

therefore

$$
\begin{aligned}
\left\|A_{0} X f\right\|^{2} & \leq k^{2}\|X f\|^{2}-\|B X f\|^{2}=k^{2}\langle X f, X f\rangle-\langle B X f, B X f\rangle \\
& =\left\langle k^{2} I X f, X f\right\rangle-\left\langle B^{*} B X f, X f\right\rangle \\
& =\left\langle\left(k^{2} I-B^{*} B\right) X f, X f\right\rangle \\
& \leq\left\|\left(k^{2} I-B^{*} B\right)^{1 / 2} X f\right\|^{2} .
\end{aligned}
$$

So we have the form

$$
\left\|A_{0} g\right\|^{2} \leq\|D g\|^{2}, g \in \mathrm{r}(X)^{-} \text {and } D=\left(k^{2} I-B^{*} B\right)^{1 / 2} .
$$

Applying Theorem E with $N=\mathrm{r}[X]^{-}, K=\mathrm{r}\left[\phi\left(E_{1}\right)\right]$ and $L=H, A_{0} \in B(N, K)$, $D \in B(H, H)$, we see that there is an operator $\widetilde{A}$ in $B\left(H, \mathrm{r}\left[\phi\left(E_{1}\right)\right]\right)$ such that $\|\widetilde{A} x\| \leq\|D x\|$ for all $x$ in $H$, and such that $\widetilde{A} X f=A_{0} X f$ for all $f$ in $H$. Finally set $A_{[n]}=\widetilde{A}+B$. It is the $A_{[n]}$ that we are looking for.

First: we have $A_{[n]} \in \mathcal{U}_{\phi[n]}$. We only need to check that $\widetilde{A}$ is in $\mathcal{U}_{\phi[n]}$.

For (i) if $E=E_{1}$, since $\widetilde{A} E_{1} H \subseteq \mathrm{r}\left[\phi\left(E_{1}\right)\right]$, then $\phi\left(E_{1}\right)^{\perp} \widetilde{A} E_{1}=0$.

For (ii) if $E=E_{j}, i=2,3, \ldots, n$, then we have

$$
\begin{aligned}
\phi\left(E_{i}\right)^{\perp} \widetilde{A} E_{i} & =\phi\left(E_{1}\right)^{\perp} \phi\left(E_{i}\right)^{\perp} \widetilde{A} E_{i} \quad\left(\text { since } E_{i}>E_{1} \text { so } \phi\left(E_{i}\right)^{\perp} \leq \phi\left(E_{1}\right)^{\perp}\right) \\
& =\phi\left(E_{i}\right)^{\perp} \phi\left(E_{1}\right)^{\perp} \widetilde{A} E_{i} .
\end{aligned}
$$

Since $\mathrm{r}\left[\widetilde{A} E_{i}\right] \subseteq \mathrm{r}\left[\phi\left(E_{1}\right)\right]$, we have $\phi\left(E_{1}\right) \widetilde{A} E_{i}=0$ and $\phi\left(E_{i}\right)^{\perp} \widetilde{A} E_{i}=0$, from which we have $\widetilde{A}$ in $\mathcal{U}_{\phi[n]}$.

Second: we have

$$
\begin{aligned}
A_{[n]} X & =\widetilde{A} X+B X=A_{0} X+B X \\
& =\phi\left(E_{1}\right)^{\perp} Y+\phi\left(E_{1}\right) Y=Y .
\end{aligned}
$$

Third: the facts $\mathrm{r}[\widetilde{A}] \subseteq \mathrm{r}\left[\phi\left(E_{1}\right)\right]$ and $\mathrm{r}[B] \subseteq \mathrm{r}\left[\phi\left(E_{1}\right)^{\perp}\right]$ mean that for any vector $x$,

$$
\begin{aligned}
\left\|A_{[n]} x\right\|^{2} & =\|\widetilde{A} x+B x\|^{2}=\|\widetilde{A} x\|^{2}+\|B x\|^{2} \leq\|D x\|^{2}+\|B x\|^{2} \\
& =\left\langle\left(k^{2} I-B^{*} B\right)^{1 / 2} x,\left(k^{2} I-B^{*} B\right)^{1 / 2} x\right\rangle+\|B x\|^{2} \\
& =\left\langle\left(k^{2} I-B^{*} B\right) x, x\right\rangle+\|B x\|^{2} \\
& =k^{2}\|x\|^{2}-\|B x\|^{2}+\|B x\|^{2}=k^{2}\|x\|,
\end{aligned}
$$

so

$$
\left\|A_{[n]}\right\| \leq k .
$$

This completes the proof for any subnest of $\mathcal{L} a t \mathcal{A}$ with $n$ nontrivial projections and by induction for all finite subnests.

The remainder of the proof is the same as [5].

Consider a maximal chain $\mathcal{M}$ of finite subnests of $\mathcal{L}$ at $\mathcal{A}$, ordered by inclusion. The net $\left\{A_{\mathcal{F}} \in \mathcal{U}_{\phi(\mathcal{F})}: \mathcal{F} \in \mathcal{M}\right\}$ is bounded (by $\left.(*)\right)$ and will therefore have a weak 
limit point, say $A$. Clearly, $A X=Y$. Furthermore, because, for any projection $E$ in $\mathcal{L} a t \mathcal{A}, E$ will eventually lie in some finite subnest in the chain $\mathcal{M}$, we have $A \in \mathcal{U}$. This completes the proof that (2) implies (1).

Example. For any $\phi$ in $H[u], \phi$ satisfies condition (2) of the above theorem but $\phi(0) \neq 0$. There need not exist an $A$ in $\mathcal{U}$ such that $A X=Y$. For example, let $\mathcal{N}$ be a nest given by $\mathcal{N}=\left\{0, E_{1}, E_{2}, \ldots, E_{n}, I\right\}$ and $n>1$. Set $\mathcal{A}=\operatorname{Alg} \mathcal{N}$; by [2] we know that $\mathcal{L} a t \mathcal{A}=\mathcal{N}$. Let $\phi(E)=E_{1}$ for all $E$ in $\mathcal{N}, Y=E_{1}$ and $X=0$. We have

$$
\sup \left\{\frac{\left\|\phi(E)^{\perp} Y f\right\|}{\left\|E^{\perp} X f\right\|} ; E \in \mathcal{N}, f \in H\right\}=0 ;
$$

therefore, $\phi$ satisfies condition (2), but for any $G$ in $B(H), G X=0 \neq E_{1}, G X \neq Y$.

\section{ACKNOWLEDGMENT}

I would like to express my great gratitude to the referee's suggestions and also to Professor Weibang Gong and Professor Jiankui Li for their helpful talks.

\section{REFERENCES}

[1] Anoussis, M., Katsoulis, E. G., and Moore, R. L., Trent, T. T., Interpolation problem for ideals in certain reflexive operator algebras, Houston J. Math., 19 (1993), 63-73.

[2] Davidson, K. R., Nest algebras, Longman Scientific \& Technical, 1988. MR 90f:47062

[3] Douglas, R. G., On majorization, factorization and range inclusion on Hilbert space, Proc. Amer. Math. Soc., 17 (1966), 413-415. MR 34:3315.

[4] Hopenwasser, A., The equation $T x=y$ in a reflexive operator algebra, Indiana University Math. Journal, 29 (1980), 121-126. MR 81c:47014]

[5] Katsoulis, E. G., Moore, R. L. and Trent, T. T., Interpolation in nest algebras and applications to operator corona theorems, J. Operator Theory, 29(1) (1993), 115-123. MR 95b:47052

[6] Knowles, G. J., On the structure of certain nest algebra modules, Can. J. Math., XXXIX, 6 (1987), 1405-1412. MR 89b:47063

[7] Lance, E. C., Some properties of Nest algebras, Proc. London Math. Soc., no.3, 19 (1969), 45-68. MR 39:3325

[8] Munch. N., Compact causal data interpolation, J. Math. Anal. Appl., no.2, 140 (1989), 407418. MR 90c: 47029

Department of Mathematics, Qufu Normal University 273165, Shan Dong, People's Republic of China

E-mail address: xiaoxiazhang@webpc.edu.cn 